\title{
A Functional Nuclear Epidermal Growth Factor Receptor, Src and Stat3 Heteromeric Complex in Pancreatic Cancer Cells
}

\author{
Soumya Jaganathan', Peibin Yue', David C. Paladino', Jelena Bogdanovic ${ }^{2,3}$, Qun Huo ${ }^{2,3}$, James \\ Turkson $^{1 *}$
}

1 Burnett School of Biomedical Sciences, College of Medicine, University of Central Florida, Orlando, Florida, United States of America, 2 NanoScience Technology Center, University of Central Florida, Orlando, Florida, United States of America, 3 Department of Chemistry, University of Central Florida, Orlando, Florida, United States of America

\begin{abstract}
Evidence is presented for the nuclear presence of a functional heteromeric complex of epidermal growth factor (EGFR), Src and the Signal Transducer and Activator of Transcription (Stat)3 proteins in pancreatic cancer cells. Stat3 remains nuclear and associated with Src or EGFR, respectively, upon the siRNA knockdown of EGFR or Src, demonstrating the resistance of the complex to the modulation of EGFR or Src alone. Significantly, chromatin immunoprecipitation (ChIP) analyses reveal the nuclear EGFR, Src and Stat3 complex is bound to the c-Myc promoter. The siRNA knockdown of EGFR or Src, or the pharmacological inhibition of Stat3 activity only marginally suppressed c-Myc expression. By contrast, the concurrent modulation of Stat3 and EGFR, or Stat3 and Src, or EGFR and Src strongly suppressed c-Myc expression, demonstrating that the novel nuclear heteromeric complex intricately regulates the c-Myc gene. The prevalence of the transcriptionally functional EGFR, Src, and Stat3 nuclear complex provides an additional and novel mechanism for supporting the pancreatic cancer phenotype and explains in part the insensitivity of pancreatic cancer cells to the inhibition of EGFR, SrC or Stat3 alone.
\end{abstract}

Citation: Jaganathan S, Yue P, Paladino DC, Bogdanovic J, Huo Q, et al. (2011) A Functional Nuclear Epidermal Growth Factor Receptor, Src and Stat3 Heteromeric Complex in Pancreatic Cancer Cells. PLoS ONE 6(5): e19605. doi:10.1371/journal.pone.0019605

Editor: Marcelo G. Bonini, University of Illinois at Chicago, United States of America

Received November 30, 2010; Accepted April 12, 2011; Published May 5, 2011

Copyright: (C) 2011 Jaganathan et al. This is an open-access article distributed under the terms of the Creative Commons Attribution License, which permits unrestricted use, distribution, and reproduction in any medium, provided the original author and source are credited.

Funding: This work was supported by the National Cancer Institute Grants CA106439 (JT) and CA128865 (JT), Florida Department of Health Bankhead-Coley Foundation (Bridge Grant) (HQ), partial support from World Gold Council (GROW Program) (HQ), and the National Natural Science Foundation of China Overseas Young Investigator Award (20828006) (HQ). The funders had no role in study design, data collection and analysis, decision to publish, or preparation of the manuscript.

Competing Interests: The authors have declared that no competing interests exist.

*E-mail: james.turkson@.ucf.edu

\section{Introduction}

Many intracellular biochemical processes are triggered by the assembly of proteins into macromolecular complexes. The association between proteins or of proteins with other molecular entities modulates protein conformation, providing a means to regulate the myriad of biochemical processes that serve to efficiently manage vital biological responses. Protein dynamics and trafficking, and protein stability are also processes that can be modulated by the association of proteins with others. In the broader sense, intermolecular associations allow specialty proteins, such as receptors, adapters, enzymes, and transcription factors to differentially modulate intracellular events, thereby creating the diversity in physiological responses and promoting context dependency.

During the induction of signal transduction, there is assembly of different proteins, each of which has specific functions important for the signal transduction and the accompanying biological response. The traditional epidermal growth factor receptor (EGFR) signal transduction pathway incorporates the activation of the mitogen-activated protein kinase kinase (MEK)-mitogenactivated protein kinase/extracellular signal-regulated kinase $\left(\operatorname{Erk}^{\mathrm{MAPK}}\right)$ and promotes mitogenic responses $[1,2]$. The EGFR induction also promotes the activation of the Signal Transducer and Activator of Transcription (STAT) family of proteins, which similarly have a central role in EGF-induced biological responses [1]. The STAT proteins are latent cytoplasmic transcription factors that are activated in response to cellular stimulation by cytokines and growth factors [3] via the phosphorylation of a critical tyrosyl residue (Tyr705 for Stat3). The tyrosine phosphorylation of STATs is mediated by tyrosine kinases of growth factor receptors and by cytoplasmic tyrosine kinases, such as Src and Janus kinase (Jaks) families. Activated STATs as dimers in the nucleus bind to specific DNA response elements in the promoters of target genes to induce gene transcription. The nuclear translocation mechanism for STATs has been the subject of recent intense investigation. Stat3 nuclear translocation has been reported to be mediated by the recognition and transport by importin- $\alpha$ and the Ran-GTPase [4], and by mechanisms involving the chaperoning by MgcRacGAP [5], EGF receptormediated endocytosis [6], and by plasma membrane-associated lipid rafts trafficking [7].

The prevalence of many hyperactive signal transduction pathways that support the cancer phenotype is a major challenge to therapy. Further to the classical way of promoting crosstalks 
among multiple signaling pathways, macro-molecular protein assemblies provide additional unique mechanisms for inducing events that would support the malignant phenotype. Such a nontraditional signaling mechanism has been identified for the EGFR, which has been detected in the cell nucleus and observed to function as a transcription factor [8,9]. Studies further revealed the nuclear EGFR complexes with Stat3 in breast cancer cells, and this complex induces specific genes, including the inducible nitric oxide synthase (iNOS) [10]. The additional EGFR function would compound its role as a mitogen and a promoter of cell survival, which all favor cancer. In that regard, the concurrent aberrant activation of EGFR and downstream signal mediators, including Src and Stat3, which occur with high frequencies in human cancers reflects an overall signaling complexity that supports the cancer phenotype. For example, with reference to pancreatic cancer, aberrant activation of EGFR occurs in $30-50 \%$ of cases [11], activated c-Src is noted in more than $70 \%$ of cases, and frequently accompanies EGFR overexpression [12], while aberrant Stat 3 activation is also highly prevalent $[13,14,15]$. Importantly, our recent report that pancreatic cancer is more sensitive to the concurrent inhibition of aberrant Stat 3 and EGFR or Src [16] shows the utilization of multiple aberrant signaling pathways for the maintenance of the cancer phenotype and how this influences the responsiveness to therapy. To extend our earlier studies [16], we sought to probe the molecular and functional interplay between Stat3, EGFR and Src and the underlying mechanisms of support of the pancreatic cancer phenotype. We herein provide evidence for a functional nuclear heteromeric EGFR, Src and Stat3 complex in pancreatic cancer cells, which promotes the induction of the c-Myc gene. Our report is the first on the identification of a nuclear EGFR, Src and Stat3 heteromeric complex that promotes the c-Myc gene induction. Understanding the dynamics of the EGFR, Src and Stat 3 molecular interactions in pancreatic cancer would provide basis to design novel effective multiple-targeted therapy approaches for pancreatic cancer.

\section{Materials and Methods}

\section{Cells and Reagents}

The human pancreatic cancer, Panc-1 and Colo-357 lines have all been previously described [16,17]. The immortalized human pancreatic duct epithelial cell (HPDEG) line was a kind gift of Dr. Tsao, OCI, UHN-PMH, Toronto) [18]. HPDEG were grown in Keratinocyte-SFM media supplemented with $0.2 \mathrm{ng}$ EGF, $30 \mu \mathrm{g}$ / $\mathrm{mL}$ bovine pituitary extract and containing antimycol. All other cells were grown in Dulbecco's modified Eagle's medium (DMEM) containing $5 \%$ iron-supplemented bovine calf serum and 100 units/ml penicillin-streptomycin.

\section{Peptide synthesis}

The Stat3 SH2 domain peptide inhibitor (SPI), FISKERERAILSTKPPGTFLLRFSESSK, the EGFR peptide motifs, pY1068 EGFR (pY1068), PEpYINQS and the pY1086EGFR (pY1086), PVpYHNQP were purchased from Peptide 2.0 (Fairfax, VA) at $>95 \%$ purity.

\section{Nuclear Extract Preparation and Gel Shift Assays}

Nuclear extract preparation from cells was carried out as previously described [17].

\section{Sub-cellular fractionation, and SDS-PAGE/Western Blot Analysis}

Western blotting analysis was performed as previously described $[19,20]$ on whole-cell lysates, and on cytosolic and membrane fractions, and on nuclear extracts. Sub-cellular fractions were prepared according standard protocol. Briefly, cells were washed using PBS, resuspended and lysed in low-salt buffer $(20 \mathrm{mM}$ HEPES (pH 7.9), $1 \mathrm{mM}$ EDTA, $1 \mathrm{mM}$ EGTA, $20 \mathrm{mM} \mathrm{NaF}$, $1 \mathrm{mM} \mathrm{Na} \mathrm{VO}_{4}, 1 \mathrm{mM} \mathrm{Na} \mathrm{P}_{2} \mathrm{O}_{7}, 1 \mathrm{mM}$ dithiothreitol, $1 \mathrm{mM}$ TLA, $0.5 \mathrm{mM}$ phenylmethylsulfonyl fluoride and $0.5 \%$ Nonidet P$40)$, and centrifuged $\left(13,000 \times \mathrm{g}, 4^{\circ} \mathrm{C}, 30 \mathrm{~s}\right)$ to obtain the cytosolic fraction. The pellet was washed three times with phosphatebuffered saline (PBS), resuspended in high-salt buffer $(420 \mathrm{mM}$ $\mathrm{NaCl}, 20 \mathrm{mM}$ HEPES (pH 7.9), $1 \mathrm{mM}$ EDTA, $1 \mathrm{mM}$ EGTA, $20 \%$ Glycerol, $20 \mathrm{mM} \mathrm{NaF}, 1 \mathrm{mM} \mathrm{Na}_{3} \mathrm{VO}_{4}, 1 \mathrm{mM} \mathrm{Na} \mathrm{P}_{2} \mathrm{O}_{7}$, $1 \mathrm{mM}$ dithiothreitol, $1 \mathrm{mM}$ TLA and $0.5 \mathrm{mM}$ phenylmethylsulfonyl fluoride), incubated at $4^{\circ} \mathrm{C}$ for $30 \mathrm{~min}$, with rocking, and centrifuged at $\left(13,000 \times \mathrm{g}, 4^{\circ} \mathrm{C}, 30 \mathrm{~min}\right)$ to obtain the nuclear fraction (supernatant). The pellet obtained was washed three times with PBS, resuspended in RIPA lysis buffer (50 mM Tris ( $\mathrm{pH} 7.4$ ), $150 \mathrm{mM}$ NaCl, $2 \mathrm{mM}$ EDTA, 1\% Nonidet P-40, 0.1\% SDS, $1 \mathrm{mM}$ TLA, and $0.5 \mathrm{mM}$ phenylmethylsulfonyl fluoride), incubated for $30 \mathrm{~min}$ at $4^{\circ} \mathrm{C}$ with rocking, and centrifuged (13,000 x g, $\left.4^{\circ} \mathrm{C}, 20 \mathrm{~min}\right)$. The supernatant was collected as the membrane fraction. Primary antibodies used are against pY845EGFR (Upstate Biotech, Millipore, Billerica, MA), pY705Stat3, Stat3, pY1068EGFR, pY1086EGFR, pY1173EGFR, EGFR, pY416Src, Src, and $\beta$-Actin (Cell Signaling Technology, Danvers, MA), and Tata-binding protein (TBP) (Santa Cruz Biotechnology, Santa Cruz, CA). The blocking peptides were purchased from the respective companies.

\section{Small-interfering RNA (siRNA) Transfection}

siRNA sequences for EGFR and Src were ordered from Dharmacon RNAi Technologies, Thermo Scientific (Lafayette, CO). Sequences used are: EGFR sense strand, 5'-GAAGGAAACUGAAUUCAAAUU-3'; EGFR antisense strand, 5'-pUUUGAAUUCAGUUUCGUUCUU-3'; control siRNA sense strand, 5' -AGUAAUACAACGGUAAAGAUU-3'; and control siRNA antisense strand, 5'-pUCUUUACGGUUGUAUUACUUU-3'. The c-Src SMARTpool siRNA reagent (NM-005417, Catalog \# M-003175-01-05) was used for Src. Transfection into cells was performed using $20 \mathrm{nM}$ of EGFR siRNA or $25 \mathrm{nM}$ of Src siRNA and $8 \mu$ l Lipofectamine RNAiMAX (Invitrogen Corporation, Carlsbad, CA) in OPTI-MEM culture medium (GIBCO, Invitrogen Corporation).

\section{Immunoprecipitation (IP), and Sequential Immunoprecipitation Studies}

These studies were performed as previously reported [21] using whole-cell lysates or nuclear extracts $(250 \mu \mathrm{g}$ total protein) and $5 \mu \mathrm{L}$ of anti-EGFR or anti-Src polyclonal antibody or the monoclonal anti-Stat3 antibody (Cell Signaling Technology). For specificity, immunoblotting analysis using anti-EGFR, anti-Src and anti-Stat 3 antibody was performed in the presence of the respective blocking peptide. Sequential IP studies were performed according to published procedures [10] with some modifications as follows: nuclear extracts, prepared as previously described [21], were subjected to a similar immunoprecipitation with respect to the first primary antibody, anti-EGFR antibody (Cell Signaling) or IgG (Santa Cruz) at $4^{\circ} \mathrm{C}$ overnight. The immunecomplex was then pelleted with $20 \mu \mathrm{L}$ protein A/G agarose beads (Santa Cruz), washed three times using wash buffer A $(0.1 \%$ SDS, $1 \%$ Triton X100, $2 \mathrm{mM}$ EDTA, $20 \mathrm{mM}$ Tris-HCl, $\mathrm{pH} 8.0)$, and then two times with wash buffer B $(0.1 \%$ SDS, $1 \%$ Triton X-100, $2 \mathrm{mM}$ EDTA, $500 \mathrm{mM} \mathrm{NaCl}, 20 \mathrm{mM}$ Tris-HCl, pH 8.0). Then the proteins were eluted with freshly prepared elution buffer (1\% SDS, $100 \mathrm{mM} \mathrm{NaHCO}$ ) and subjected to the second immunoprecipitation by incubating with anti-Src antibody or IgG (Santa Cruz). 
The complexes were then precipitated, washed, eluted with lamelli buffer and then subjected to SDS-PAGE and Western blotting analysis probing for Stat3.

\section{Immunostaining with laser-scanning confocal imaging}

Panc-1 cells growing on coverslips in 12-well plates were treated with or without inhibitors for 1 or $24 \mathrm{~h}$ and subjected to immunostaining and fluorescence or laser-scanning confocal microscopy, as previously described [21]. Cells were fixed with 4\% paraformaldehyde for $15 \mathrm{~min}$, washed three times with PBS, permeabilized with $0.25 \%$ Triton X-100 for $10 \mathrm{~min}$, and washed three times with PBS. Specimens were then blocked in $0.1 \%$ BSA in PBST for $30 \mathrm{~min}$ and incubated overnight at $4^{\circ} \mathrm{C}$ with rat monoclonal anti-EGFR (Santa Cruz), mouse monoclonal anti-Src (Cell Signaling), and rabbit polyclonal anti-Stat3 (Cell Signaling) antibodies at 1:50 dilution (in 0.1\% BSA). Subsequently, cells were rinsed three times in PBST, incubated for $1 \mathrm{~h}$ at room temperature in the dark with 1:1000 dilutions of the three AlexFluor secondary antibodies, ALexaFLuor405 (goat antimouse), AlexaFluor488 (donkey anti-rabbit) and AlexaFluor546 (goat anti-rat) (Molecular Probes, Invitrogen) for EGFR, Src and Stat3 detection, respectively. Specimens were then washed three with PBST. Subsequently, coverslips were removed and mounted on slides using Fluoromount-G (Southern Biotech, Birmingham, $\mathrm{AL})$ and prevented from drying by sealing the edges with nail paint. Slides were stored in the dark at $4^{\circ} \mathrm{C}$ until images were captured. For negative staining, secondary antibodies were added without the primary antibodies. Confocal analysis was performed by the examination of slides under Leica TCS SP5 confocal microscope (Germany) at appropriate wavelengths. Images were captured and processed using the Leica TCS SP 5 software.

\section{Chromatin Immunoprecipitation (ChIP) and Sequential ChIP Analyses}

For ChIP assay, cells in culture were treated with formaldehyde at a final concentration of $1 \%$, for $10 \mathrm{~min}$ at room temperature followed by treatment with glycine at a final concentration of $0.125 \mathrm{M}$ for $5 \mathrm{~min}$ at room temperature for cross-linking. Subsequently, cells were washed with ice-cold PBS and resuspended in and lysed with lysis buffer (20 mM HEPES, pH 7.4, 1 mM EDTA, 1 mM EGTA, $1 \mathrm{mM} \mathrm{NaF}, 1 \mathrm{mM} \mathrm{Na}_{3} \mathrm{VO}_{4}, 1 \mathrm{mM} \mathrm{Na}_{4} \mathrm{P}_{2} \mathrm{O}_{7}, 1 \mathrm{mM}$ dithiothreitol), 1X TLA, $1 \mathrm{mM}$ phenylmethylsulfonyl fluoride, $5 \%$ Nonidet P-40, and centrifuged. Then nuclear pellet was resuspended in nuclei lysis buffer (50 mM Tris-HCl, pH 8.0, 10 mM EDTA, 1\% SDS and protease inhibitors) (Roche, Indianapolis, IN). The nuclear lysates were sonicated (Omni International, Kennesaw, GA) at 30\% power for 3 pulses for $10 \mathrm{~s}$ intervals on ice to shear DNA. The chromatin solution was pre-cleared with protein $\mathrm{A} / \mathrm{G}$ agarose beads (Santa Cruz) for $1 \mathrm{~h}$ at $4^{\circ} \mathrm{C}$ with rocking. Then the pre-cleared lysates were immunoprecipitated by incubating with anti-EGFR, anti-Src, or antiStat 3 antibodies or with IgG (for no antibody) (Santa Cruz) at $4^{\circ} \mathrm{C}$ overnight with rocking. The complexes were collected with $20 \mu \mathrm{L}$ protein $\mathrm{A} / \mathrm{G}$ agarose beads (Santa Cruz), washed three times using wash buffer A $(0.1 \%$ SDS, $1 \%$ Triton X-100, 2 mM EDTA, $20 \mathrm{mM}$ Tris-HCl, $\mathrm{pH}$ 8.0) and two times with wash buffer B (0.1\% SDS, $1 \%$ Triton X-100, 2 mM EDTA, $500 \mathrm{mM} \mathrm{NaCl,} 20 \mathrm{mM}$ Tris-HCl, $\mathrm{pH}$ 8.0). Then complexes were eluted with freshly prepared elution buffer ( $1 \%$ SDS, $100 \mathrm{mM} \mathrm{NaHCO}$ ). Cross-links were reversed by heating at $65^{\circ} \mathrm{C}$ in the presence of $\mathrm{NaCl}$ followed by proteinase $\mathrm{K}$ treatment $(20 \mu \mathrm{l}$ of a $20 \mathrm{mg} / \mathrm{ml})$ for $6 \mathrm{~h}$. The DNA was recovered and purified using DNA purification kit from Qiagen (Valencia, CA). The purified chromatin immunoprecipitated DNA was next used as a template for the polymerase chain reaction (PCR) amplification of the c-Myc promoter using the primers, Forward, 5'AAAAGGGGAAA-
GAGGACCTGG-3', and Reverse, 5'-TAAAAGGGGCAAGTGGAGAGC-3' or the TWIST promoter using the primers, Forward, 5'- AGTCTCGTCGGACCGCTTCGTG -3'.

Reverse: 5' - CTCGGTGCAGGCGGAAAGTTTGG -3' (Invitrogen). The PCR products, $133 \mathrm{bp}$ for c-Myc and the 332-bp for TWIST [22] were resolved on $2 \%$ agarose gel. The sequential ChIP studies were performed as previously reported [10] and following the sequential immunoprecipitation studies described, using the first primary antibody (anti-EGFR) and the second primary antibody (anti-Src). The recovered complexes after the secondary immunoprecipitation were eluted with the elution buffer and then subjected to ChIP assay, as described.

\section{Gel filtration chromatography}

The pre-packed Superdex 200 10/30 GL glass column was purchased from GE Healthcare Life Sciences (Piscataway, NJ). The chromatography system used in the study was the BioLogic Duoflow System (Bio-Rad, Hercules, CA). The chromatography analysis was performed following the manufacturer's instructions with a general run sequence of equilibration, load, elution, regeneration and storage. RIPA Buffer $(50 \mathrm{mM}$ Tris, $\mathrm{pH} 7.4$, $150 \mathrm{mM} \mathrm{NaCl}, 2 \mathrm{mM}$ EDTA, $0.1 \%$ SDS, 1\% Nonidet P-40) was used as the mobile phase buffer. Samples (Panc-1 cell lysate, $2 \mathrm{mg}$ total protein in a volume of $250 \mu \mathrm{l}$ ) were loaded unto the column, then flow rate was adjusted to $0.25 \mathrm{ml} / \mathrm{min}$, and then fraction $(500 \mu \mathrm{l})$ collection was initiated right after the sample loading and monitored by the eluent absorbance at $280 \mathrm{~nm}$. According to the absorbance peaks, fractions 21-34 were selected and subjected to immunoblotting analysis using antibodies against EGFR, Stat3, and Src (Cell Signaling), and against RNA helicase A (RHA) (Abcam, Cambridge, MA). For immunoprecipitation assay, $100 \mu \mathrm{l}$ each of fractions 23-27 were pooled, from which EGFR immunecomplex was precipitated using anti-EGFR antibody (Cell Signaling) and subjected to immunoblotting analysis for Stat3, Src and EGFR.

\section{Preparation of anti-EGFR and mouse IgG1-GNP probes}

Gold nanoparticles (GNPs), $0.1 \mathrm{nM}$, with a diameter of $40 \mathrm{~nm}$ were purchased from Ted Pella Inc. (Redding, CA). Mouse monoclonal anti-EGFR [F4] antibody was purchased from Abcam (cat. no. ab62, conc. $1.2 \mathrm{mg} / \mathrm{ml}$ ), and non-specific mouse monoclonal IgG1 was purchased from Sigma (cat. no. M9629, conc. $1 \mathrm{mg} / \mathrm{ml}$ ). Polyclonal anti-Stat3 (conc. $0.2 \mathrm{mg} / \mathrm{ml}$ ), polyclonal antiSrc $(0.1 \mathrm{mg} / \mathrm{ml})$, polyclonal anti-EGFR $(0.2 \mathrm{mg} / \mathrm{ml})$ and nonspecific rabbit IgG $(0.4 \mathrm{mg} / \mathrm{ml})$ were purchased from Santa Cruz. All other chemicals and buffer ingredients for the assay development were purchased from Sigma. The anti-EGFR-GNP probe was prepared by adding $10 \mu \mathrm{l}$ of mouse monoclonal anti-EGFR antibody to $1 \mathrm{ml}$ of GNPs. After incubation for $15 \mathrm{~min}$ at room temperature, the probe was blocked with $2.5 \mathrm{mg}$ BSA for $30 \mathrm{~min}$. After the centrifugation at 10,000 rpm for $5 \mathrm{~min}$, the supernatant was discarded and the nanoparticle residue was redispersed in $0.5 \mathrm{ml}$ of $0.25 \% \mathrm{BSA}$ in $10 \mathrm{mM}$ phosphate buffer $(\mathrm{PB})$. The probe was then used in the assay. The negative control mouse IgG1-GNP probe was prepared by adding $10 \mu \mathrm{l}$ of mouse monoclonal IgGl antibody to $1 \mathrm{ml} \mathrm{GNPs}$, and following the procedure identical to the one used for the EGFR probe preparation. Mouse IgGl was used here to prepare the control probe, because the anti-EGFR monoclonal antibody is a mouse IgGl type antibody.

\section{Detection and kinetic binding study of EGFR from nuclear extract to the GNP probes}

The Panc-1 nuclear extract sample was diluted in phosphate buffer $(\mathrm{PB})$ to $1 \mathrm{mg} / \mathrm{ml}$ of total protein. In a sample cell for 
Dynamic Light Scattering (DLS) measurement (Hellma cuvette QS $3 \mathrm{~mm}), 20 \mu \mathrm{l}$ of the anti-EGFR-GNP probe was mixed with $2 \mu \mathrm{l}$ of the sample, and the particle size increase was read with a DLS instrument (Zetasizer Nano ZS90 DLS system, Malvern Instruments Ltd, England) at exactly 1, 6, 11, 16, and 30 min after the mixing. The same experiment was also performed using the mouse IgGl-GNP probe. In order to confirm the specificity of the anti-EGFR-GNP probe in the detection of EGFR from nuclear extract, an inhibition experiment was conducted by treating $5 \mu \mathrm{l}$ of the sample with $1 \mu \mathrm{l}$ of monoclonal anti-EGFR antibody at room temperature for $7 \mathrm{~min}$ and $24 \mathrm{~min}$ prior to using the sample in the assay. After this incubation, $20 \mu \mathrm{l}$ of the anti-EGFR-GNP probe was mixed with $2 \mu \mathrm{l}$ of the treated sample, and the particle size was read at 1, 6 and 11 min after the mixing.

\section{Protein complex binding partner study using polyclonal antibody}

In a $1.5 \mathrm{ml}$ microcentrifuge tube, $80 \mu \mathrm{l}$ of the anti-EGFRGNP probe was mixed with $8 \mu \mathrm{l}$ of the sample. After incubation for $30 \mathrm{~min}$ at room temperature, this solution was divided into four $20 \mu \mathrm{l}$ portions. After transferring into the sample cell, the particle size of each of these portions was read with a DLS instrument. After this reading, the solution was spiked with a polyclonal antibody: either with $1 \mu \mathrm{l}$ of anti-Stat 3 or $2 \mu \mathrm{l}$ of anti-Src or $1 \mu \mathrm{l}$ of anti-EGFR or $0.5 \mu \mathrm{l}$ of rabbit IgG. The particle size increase was read at exactly $5 \mathrm{~min}$ and $10 \mathrm{~min}$ after the start of the first reading.

\section{Dynamic Light Scattering (DLS) measurements}

The DLS measurements of all sample solutions were conducted using a Zetasizer Nano ZS90 DLS system equipped with a red (633 nm) laser and an Avalanche photodiode detector (APD) (quantum efficiency $>50 \%$ at $633 \mathrm{~nm}$ ) (Malvern Instruments Ltd). DTS applications 5.10 software was used to analyze the data. The average particle size (Z-average) of the solution was obtained using a Cumulant method. For each sample, ten DLS measurements were conducted with one run, and each run lasted for 10 seconds. All measurements were done at a $90^{\circ}$ detection angle.

\section{Results}

Detection of nuclear EGFR, Src and Stat3 heterocomplex

We sought to investigate the signaling complex assembly and the dynamics of the interactions of the hyperactive Stat3, EGFR and Src $[14,16,23]$ within the context of the human pancreatic cancer phenotype. Co-immunoprecipitation (co-IP) with immunoblotting analysis shows, EGFR immunecomplex from Panc-1 or Colo-357 whole-cell lysates contained both Src and Stat3 (Fig. 1A(i)), Src immunecomplex contained both EGFR and Stat3 (Fig. 1A(ii)), while Stat3 immunoprecipitate contained EGFR and Src (Fig. 1A(iii)). For specificity, the non-specific rabbit IgG in the immunoprecipitation and immunoblotting analysis for Src, Stat3 or EGFR showed no detectable protein (Fig. 1A, IgG, and data not shown), and the immunoblotting analysis performed on the immune precipitates in the presence of the respective blocking peptides (BP) showed a complete or near complete block of the immune detection of Stat3, Src or EGFR, compared to the levels detected in the absence of the blocking peptides (Fig. 1B(i)-(iii), compare + BP, lower panels to - BP, upper panels). We determined the effect of siRNA knockdown of EGFR or Src on the complex formation. Co-immunoprecipitation with immunoblotting studies of whole-cell lysates from Panc-1 cells showed that when EGFR is knockdown by siRNA (Fig.1C(i), upper band), Src immunecomplex remains associated with Stat3 (Fig. 1C(i), IP:Src), and vice-versa (Fig. 1C(i), IP:Stat3). Likewise, when $\mathrm{Src}$ is knockdown by siRNA (Fig. 1C(ii), upper band), EGFR immunecomplex remains associated with Stat3 (Fig. 1C(ii), IP:EGFR), and vice-versa (Fig. 1G(ii), IP:Stat3). Scrambled (con) siRNA has no effect. These findings indicate that with respect to the EGFR, Src and Stat 3 heteromeric complex, Stat3 proteins remains associated with Src or EGFR, respectively, upon the siRNA knockdown of EGFR or Src.

We asked the question whether the observed EGFR, Src and Stat3 heteromeric complex was present in the nucleus. Coimmunoprecipitation with immunoblotting analysis of nuclear extracts shows that EGFR immunecomplex contained both Stat3 and Src (Fig. 2A(i), IP:EGFR), Src immunecomplex contained both Stat3 and EGFR (Fig. 2A(ii), IP:Src), while Stat3 immunecomplex contained both EGFR and Src (Fig. 2A(iii), IP:Stat3). These data demonstrated the presence of the EGFR, Src and Stat3 heteromeric complex in the nucleus. For specificity of the immunoreagents, the non-specific rabbit IgG pull-down samples that were similarly immunoblotted showed no detectable EGFR, Src or Stat3 (Fig. 2A, IgG and data not shown). Immunoblotting analysis probing for the Tata-binding protein (TBP) confirmed that the extracts used in these studies are of nuclear origin (Fig. 2A(iv)). The heteromeric complex was validated by performing sequential immunoprecipitation analysis, whereby EGFR immunecomplex (IP:EGFR) was further subjected to a secondary immunoprecipitation using anti-Src antibody (IP:EGFR/IP:Src) and then immunoblotted for EGFR and Stat3. The results of these studies showed the presence of EGFR and Stat3 in the sequential immunoprecipitates (Fig. 2B, IP:EGFR/ IP:Src). By contrast, IgG pull-down that was subjected to immunoblotting for EGFR or Stat3 showed no detectable levels (Fig. 2B, IgG), further confirming the specificity of the immunoreagents used.

The presence of the EGFR, Src and Stat3 complex in the nucleus of pancreatic cancer cells was further investigated by subjecting nuclear extract preparations to gel filtration column chromatography analysis (Superdex200, exclusion limit $200 \mathrm{kD}$ ), as described in "Materials and Methods", in conjunction with immunoblotting analysis. The collected fractions, which showed peak absorbance at $280 \mathrm{~nm}$ (data not shown) were immunoprobed. Results showed that the EGFR protein first appears in the fraction 23 and is present together with Stat3 and Src, and the three proteins are concurrently detected in the fractions 23-29 (Fig. S1A). Results also showed detectable Stat3 and Src proteins in fractions 30 through 32 well after EGFR was completely eluted (Fig. S1A). Analysis of the previously-reported EGFR protein partner, RNA helicase A (RHA) [24] also showed detectable levels that were predominantly in the fractions 23 and 24 (Fig. S1A, RHA). These fractions were further subjected to co-immunoprecipitation analysis to validate the presence of the complex. Immunoblotting analysis of EGFR immunecomplex prepared from the pooled fractions 23-27 further showed the presence of Src and Stat3 (Fig. S1B). The early and the concurrent elution of EGFR, Src and Stat 3 raise the possibility that each of the proteins is part of a higher molecular weight protein complex, and also that the three proteins associate as part of the same complex, as suggested by the immunecomplex formation. There has been a previous report about nuclear EGFR/Stat3 complex under conditions of cellular stimulation by EGF [10]. We therefore investigated whether the nuclear EGFR, Src, Stat3 complex was present constitutively (without ligand stimulation) in tumor cells. Immunoblotting analysis did not detect any appreciable levels of Stat3 or Src in EGFR immunecomplex or of Src or EGFR in Stat3 immunoprecipitate from the nuclear extracts prepared from 
A

(i)

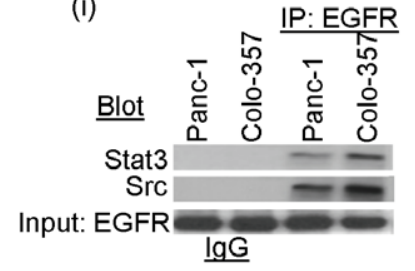

(ii)

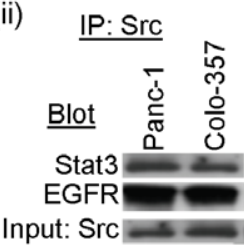

(iii)

(iii) IP: Stat3

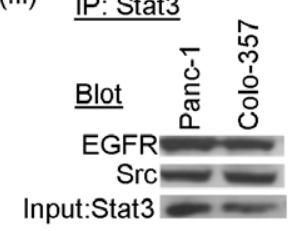

(ii) $\underline{\mathrm{WB}}$
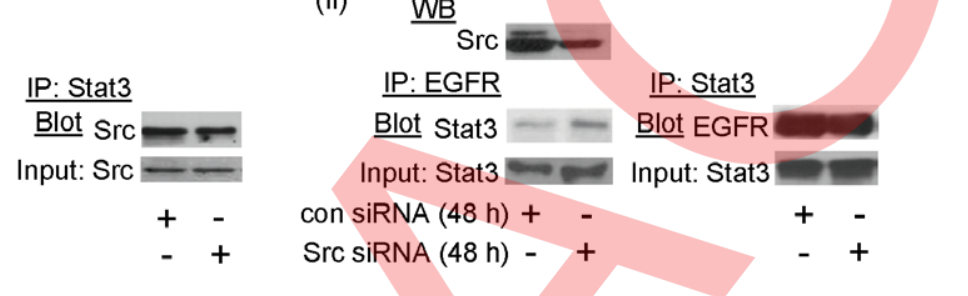

(i)

(i) WW

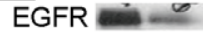

IP: SrC

Blot Stat3 9

Input: Stat $3=$

EGFR SiRNA (48h) - + (iii)
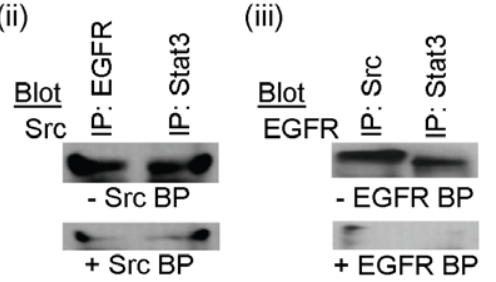

+ EGFR BP

Figure 1. Co-immunoprecipitation with immunoblotting analysis of EGFR, Src and Stat3 association in Panc-1 and Colo-357 cells. Immunoblotting analyses of immunecomplexes of EGFR (IP:EGFR), Src (IP:Src), and Stat3 (IP:Stat3), or of non-specific IgG non-immunoprecipitate prepared from whole-cell lysates of Panc-1 or Colo-357 cells untransfected (A and B) or transfected with EGFR siRNA, Src siRNA, or control (con) siRNA (C) and probing for Src, Stat3 and EGFR in the absence (A and C) or presence (B) of Stat3 blocking peptide (Stat3 BP), Src blocking peptide (Src BP) or EGFR blocking peptide (EGFR BP). Bands corresponding to proteins in gel are shown; input: except where indicated, represents the immunoblotting for the respective immunoprecipitated protein in the same amount of lysate used in the assay; Data are representative of 3 independent studies. doi:10.1371/journal.pone.0019605.g001

human breast cancer, MDA-MB-231 and the human non-small cell lung cancer, A549 lines (Fig. S1C), suggesting a minimal possibility of the existence of a constitutive nuclear EGFR, Src, Stat3 complex in the two cancer cell types. These studies together show for the first time that EGFR, Src and Stat3 form a heteromeric complex in the nucleus of pancreatic cancer cells.

Given the detection of the EGFR, Src and Stat 3 complex in whole-cell and nuclear lysates, we were interested to determine the relative levels and the sizes of EGFR, Src and Stat3 in the different sub-cellular fractions. Membrane and cytosolic fractions, and nuclear extracts were prepared from Panc-l cells according to established protocols and which involved using $10 \%$ Nonidet P-40 lysis, and a low-salt HEPES buffer extraction for cytosolic extract, a high-salt HEPES buffer extraction for nuclear extracts $(18,22)$, and $0.5 \%$ SDS buffer for the membrane fraction. Immunoblotting analysis of samples of equal total protein from the sub-cellular fractions shows that with respect to each of the EGFR, Src, or Stat3 protein, the size is the same in the membrane (Mem), cytosolic (Cyto), or nuclear fraction (Nuc) (Fig. 2C). Results further show that the level of the total EGFR protein is highest in the membrane, and is higher in the cytosolic fraction than in the nuclear extract (Fig. 2C (i)). By contrast, results show that Stat3 levels are highest in the cytosolic fraction, and higher in the nuclear extract, compared to the levels associated with the cell membrane (Fig. 2C(ii)). The results for Src also showed noticeable differences, with the membrane-associated levels higher than both the cytosolic and nuclear levels, which were nearly identical (Fig. 2C(iii)). Significantly, the non-denaturing conditions $(10 \%$ Nonidet P-40, low- or high-salt extraction) for preparing the cytosolic and nuclear extracts raises the potential that the EGFR protein detected in the cytosolic compartment and in the nucleus is a soluble form, and further that the Stat3 protein in the cytosolic fraction and in the nucleus is potentially a dimer. Moreover, this is the first report of the nuclear presence of $\mathrm{c}-\mathrm{Src}$ tyrosine kinase in cancer cells.

The role of EGFR and Src tyrosine kinases in the nuclear EGFR, Src and Stat3 heterocomplex formation

We determined the importance of EGFR or Src in the assembly of the nuclear complex by examining the effect of siRNA knockdown. Immunoblotting analysis of immunecomplexes of Stat3 (IP:Stat3), EGFR (IP:EGFR) or Src (IP:Src) prepared from nuclear extracts showed that the siRNA knockdown of Src (Fig. 3A(i), upper panel) has little effect on the association of Stat3 with EGFR in the nucleus (Fig. 3A(i), IP:Stat3, IP:EGFR), while similarly, the knockdown of EGFR (Fig. 3A(ii), upper panel) did not alter the binding of Stat3 to Src (Fig. 3A(ii), IP:Stat3, IP:Src). Scrambled (con) siRNA has no effect. Similar to the results for the 
A Nuclear extract (i)

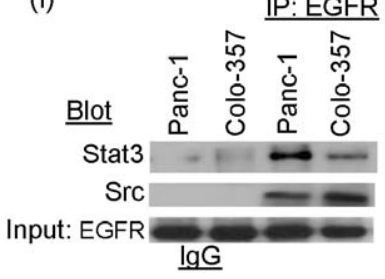

(ii)

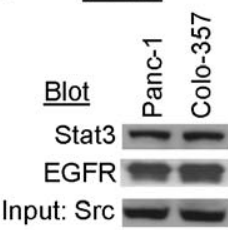

(iii)

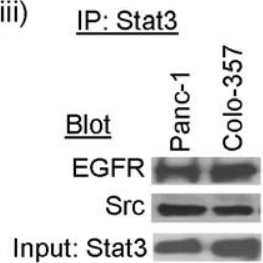

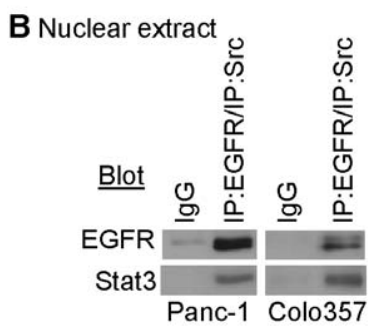

C Sub-cellular fractions

(i)

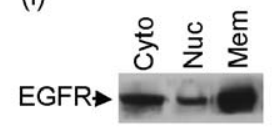

(ii)

$\underline{\text { Panc-1 }}$

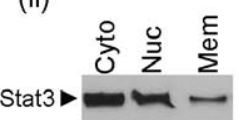

(iii)

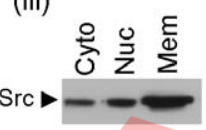

(iv)
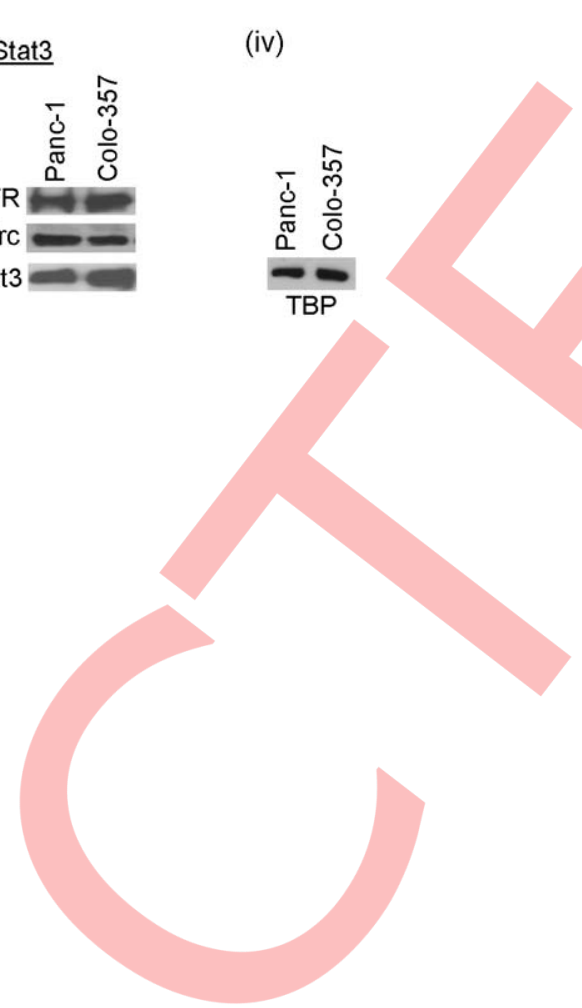

Figure 2. Co-immunoprecipitation with immunoblotting analysis of EGFR, Src and Stat3 complex in the nucleus and the subcellular distribution of EGFR, Src and Stat3. (A and B) Immunoblotting analyses of immunecomplexes of EGFR (IP:EGFR), Src (IP:Src), Stat3 (IP:Stat3), EGFR/Src (IP:EGFR/IP:Src), or of non-specific IgG non-immuneprecpitate prepared from nuclear extracts of Panc-1 or Colo-357 cells and probing for Stat3, EGFR, Src, or the Tata-binding protein (TBP); and (C), immunoblotting analysis of membrane (mem) and cytosolic (cyto) fractions and of nuclear (nuc) extracts from Panc-1 cells probing for (i) EGFR, (ii) Stat3 and (iii) Src. Bands corresponding to proteins in gel are shown; input: except where indicated, represents the immunoblotting for the respective immunoprecipitated protein in the same amount of nuclear extract used in the assay; IP:EGFR/IP:Src, sequential immunoprecipitation with anti-EGFR and then anti-Src antibody; Data are representative of 3 independent studies.

doi:10.1371/journal.pone.0019605.g002

whole-cell lysates analysis in Figure $1 \mathrm{C}$, these data together indicate that the knockdown of one protein does not preclude the interaction between the other two protein partners.

We next sought to determine if the heteromeric complex formation is dependent on the tyrosine kinase activities of EGFR and Src. Compared to IgG, immunoblotting of immunecomplex of EGFR showed no effect of 1 h-treatment of Panc-1 cells with the selective EGFR inhibitor, Iressa (ZD1839, ZD), the Src inhibitor, Dasatinib (Das), or the Stat3 dimerization disrupting inhibitor, S3I-201 [25] (Fig. 1G, IP:EGFR, lanes 3, 6, and 8, compared to lane 2). By contrast, immunoblotting analysis of EGFR immunecomplex showed decreased levels of associated Stat3 protein, but not Src, when cells were treated with ZD, Das, or S3I-201 for $24 \mathrm{~h}$ (Fig. 3B, lanes 4, 7, and 9). These findings together suggest the EGFR and Src kinase activities promote the association of Stat3 with EGFR in the nuclear heteromeric complex, while the inhibition of EGFR or Src kinase alone does not preclude the interaction between EGFR and Src. Furthermore, the Stat3 dimerization disrupting inhibitor blocks Stat3 association in the complex; however, the disruption of Stat3 dimerization, and hence its binding to EGFR does not preclude EGFR interaction with Src. The observation that the nuclear heteromeric EGFR, Src, and Stat3 complex was not completely dissociated and that EGFR/Src complex persisted by 24-h inhibition of EGFR (ZD) or Src (Das) (Fig. 3B, lanes 4, and 9) has important clinical implications in regard to the responsiveness of pancreatic cancer cells to a monotherapy targeting EGFR or Src.

During the stimulation of the EGFR signaling pathway, key phospho-tyrosine $(\mathrm{pY})$ peptide motifs are induced, which recruit different signaling proteins, including Stat3 [26,27]. The Stat3 $\mathrm{SH} 2$ domain binds to the receptor $\mathrm{pY}$ motifs through $\mathrm{pY}-\mathrm{SH} 2$ domain interactions [28]. We therefore sought to probe further the observed nuclear complex of the EGFR, Src and Stat3 with respect to the peptide motifs potentially involved in the interactions by using the known Stat3-binding EGFR motifs, pY1068 peptide and pY1086 peptide, and the newly reported Stat3 peptide inhibitor, SPI, which is derived from the Stat3 $\mathrm{SH} 2$ domain and is capable of blocking the binding of the Stat3 $\mathrm{SH} 2$ domain to $\mathrm{pY}$ peptide motifs [29]. Immunoblotting analysis of Stat3 immunoprecipitates from nuclear extracts that have been pre-incubated with the three peptides for $2 \mathrm{~h}$ at room temperature showed no detectable EGFR protein (Fig. 3G(i)). These results suggest the EGFR-Stat3 interaction is promoted by pY1068EGFR and pY1086EGFR, and also that in binding to EGFR, Stat3 utilizes the key amino acid residues, 588-615 of its SH2 domain, which make up the peptide inhibitor SPI [29]. By contrast, similar probing with the SPI peptide showed no significant effect on the immuno-detection of Src protein within the Stat3 immunecomplex (Fig. 3G(ii)), suggesting the direct interaction between Src and Stat3 is independent of the amino acid residues 588-615 of the Stat3 SH2 domain. 
A
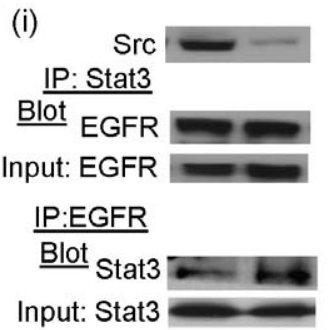

(ii)

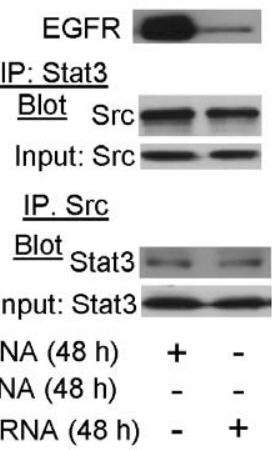

C

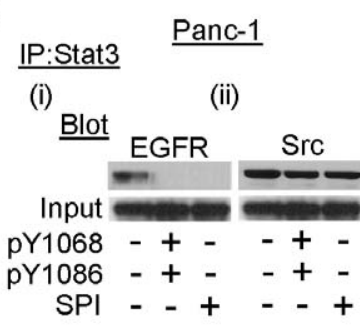

$\mathrm{SPI}-+-+$

B

$\begin{array}{lllll}+ & - & \text { Con siRNA }(48 \mathrm{~h}) & + & - \\ - & + & \text { Src siRNA }(48 \mathrm{~h}) & - & - \\ - & - & \text { EGFR SiRNA }(48 \mathrm{~h}) & - & +\end{array}$

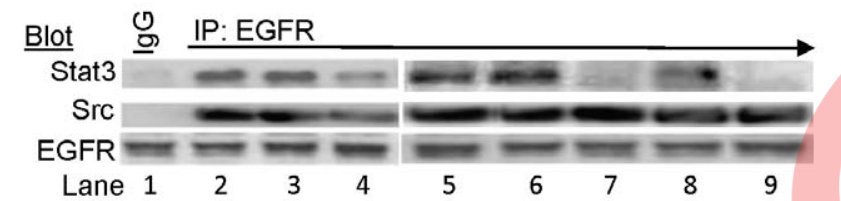

Lane $121 \quad 3 \quad 4$

$1 \mu \mathrm{M} Z \mathrm{D}(\mathrm{h})-\quad-\quad 124$

$50 \mu \mathrm{M}$ S3I-201 (h) -

$100 \mathrm{nM}$ Das $(\mathrm{h})$ -

Figure 3. Co-immunoprecipitation with immunoblotting analysis of the effects of modulation of EGFR, Src and Stat3 on the nuclear EGFR, Src and Stat3 complex. (A, B, and C) Immunoblotting analyses of immunecomplexes of Stat3 (IP:Stat3), EGFR (IP:EGFR), or Src (IP:Src) prepared from nuclear extracts of Panc-1 cells untransfected or transfected with Src siRNA, EGFR siRNA, or control (con) siRNA (A), or treated with or without the EGFR inhibitor (ZD1839, ZD), Src inhibitor (Dasatinib, Das), or the Stat3 inhibitor (S3I-201) for 1 or $24 \mathrm{~h}$ (B), or from nuclear extracts preincubated for $2 \mathrm{~h}$ with or without $100 \mu \mathrm{M}$ pY1068, pY1086, or SPI peptide (C) and probing for EGFR, Src, Stat3; or (D) immunoblotting analysis of nuclear extracts prepared from Panc-1 cells treated or untreated with phenylarsine oxide (PAO) and probing for Src, Stat3, EGFR. Bands corresponding to proteins in gel are shown; input: except where indicated, represents the immunoblotting for the respective immunoprecipitated protein in the same amount of lysate or nuclear extract used in the assay; Data are representative of 3 independent studies.

doi:10.1371/journal.pone.0019605.g003

Nuclear EGFR, Src and Stat3 heterocomplex is independent of EGFR-mediated endocytosis

We were interested to determine how the known nuclear translocation mechanisms might affect the nuclear presence of the heteromeric EGFR, Src and Stat3 complex. Several mechanisms have been reported for EGFR nuclear translocation, including receptor endocytosis, endosomal sorting machinery, importins alphal/betal, and exportin CRM1 [30,31]. Previous studies have also shown that Stat3 nuclear translocation is facilitated by EGFR-mediated endocytosis [6]. However, the siRNA knockdown of EGFR did not affect the nuclear presence of Src/Stat3 (Fig. 3A(ii)), suggesting EGFR-mediated mechanisms may not be utilized for the nuclear localization of Stat3 or Src. To further determine whether endocytosis is required for the nuclear presence of the heteromeric complex, cells were treated with the pharmacological inhibitor of endocytosis, phenylarsine oxide [6]. In contrast to PAO-induced inhibition of nuclear Stat3 DNA-binding activity induced by EGF [6], immunoblotting analysis showed treatment with PAO has no effect on the nuclear presence of EGFR, Src and Stat3 heterocomplex in pancreatic cancer cells (Fig. 3D), suggesting the nuclear presence of the heteromeric complex is not dependent on endocytosis. Altogether, these findings show that EGFR, Src and Stat3 associate into a complex in a manner where all three proteins interact. However, our study does not exclude the possibility that other proteins could be present in the complex.
Detection and Analysis of the EGFR, Src and Stat3 heterocomplex through Nanoparticle Sizing (DANS) technology with Dynamic Light Scattering

The EGFR, Src and Stat3 association was further probed using DANS (Detection and Analysis through Nanoparticle Sizing) technology [32,33,34,35,36,37]. The principle of DANS technology for protein complex detection and binding partner analysis is a single-step solution immunoassay based on gold nanoparticle (GNP) immunoprobes coupled with dynamic light scattering (DLS) detection. Details of this technology are provided in "Text S1" and illustrated in Fig. S2. Based on this general principle, the assay is designed to detect and identify protein binding partners of a protein complex. The kinetic binding assay revealed and confirmed that the anti-EGFR-GNP probe detects EGFR from the sample specifically (Fig. 4A(i)). The increase in particle size obtained with the antiEGFR-GNP probe was much larger than the one obtained with the non-specific mouse IgGl control probe (Fig. 4A(i)). The approximate net increase of the average nanoparticle size of the assay solution was about $70 \mathrm{~nm}$, after deducting the size increase caused by non-specific interactions. For the mouse IgG1-GNP probe, the particle size increase was less than $10 \mathrm{~nm}$ after incubation with the sample for $30 \mathrm{~min}$. Furthermore, the steepness of the kinetic curve for the EGFR probe suggested specific interactions in the assay system, while the shallow curve for the mouse IgGl probe indicated the small size increase of the nanoparticle probes was due to nonspecific interactions. To further confirm the specificity of anti- 

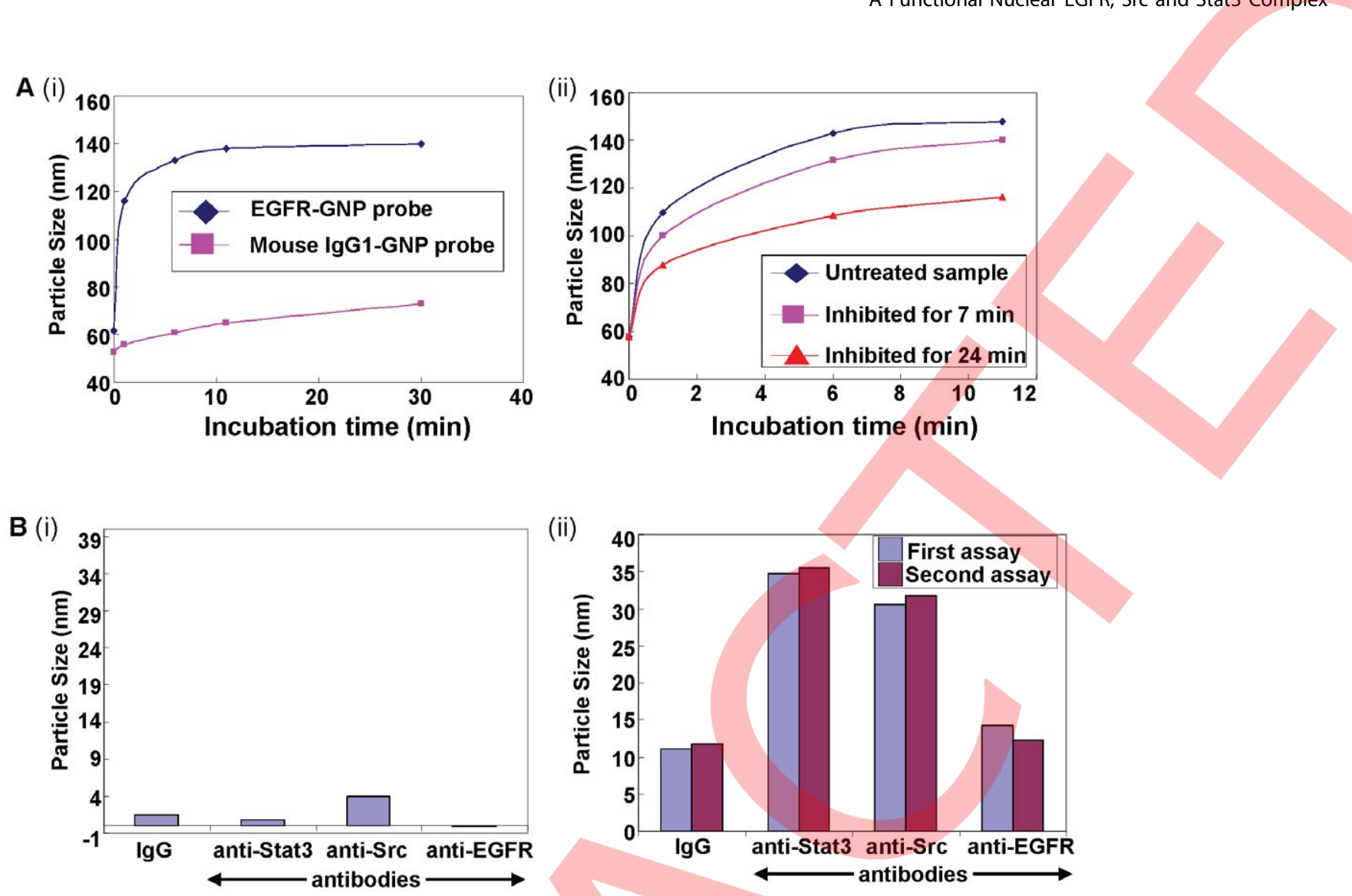

Figure 4. Studies of protein complex and protein binding partners using the Detection and Analysis through Nanoparticle Sizing technology. (A) Kinetic binding assay of EGFR-gold nanoparticle (GNP) probe (or mouse IgG1-GNP probe as negative control) binding to (i) EGFR protein and its complex from Panc-1 nuclear extracts, and the (ii) inhibitory effect of the mouse monoclonal anti-EGFR antibody on the EGFR-GNP probe binding to the EGFR protein; and (B) Protein complex binding partner analysis whereby the polyclonal anti-Stat3, anti-Src or anti-EGFR antibody or the non-specific rabbit IgG (negative control) is added to the assay solution prepared from the (i) non-specific mouse IgG1-GNP probe (negative control), or (ii) anti-EGFR-GNP probe; Data are representative of 4 independent studies. doi:10.1371/journal.pone.0019605.g004

EGFR-GNP probe in the detection of EGFR from nuclear extract, an inhibition experiment was conducted in which the extract was first treated with the monoclonal anti-EGFR antibody. It is expected that the binding between the monoclonal antibody and the EGFR protein from the sample will block the subsequent binding of EGFR (in the sample) to the GNP immunoprobes, therefore, leading to decreased nanoparticle size increase during the kinetic assay. Data shown in Figure 4A(ii) confirmed the inhibitory effect of the monoclonal anti-EGFR antibody. The magnitude of this inhibition was correlated to the sample treatment time: the 24min-treatment inhibited the particle size increase in the assay stronger than the 7 min-treatment (Fig. $4 \mathrm{~A}(\mathrm{ii})$ ).

After using the anti-EGFR-GNP probe to "catch" the EGFR protein or protein complex from the nuclear extract sample, a second polyclonal antibody (anti-Stat3, anti-Src, or anti-EGFR antibody, or non-specific rabbit polyclonal IgG) was then added to the assay solution to identify the binding partner of the complex. In negative control studies using the mouse IgGl-GNP probe, particle size remains nearly unchanged after the addition of the four polyclonal antibodies (Fig. 4B(i)). This result confirmed the kinetic binding study that the non-specific mouse IgG1-GNP probe did not bind with the EGFR protein or protein complex from the sample. The addition of a polyclonal antibody itself to the assay solution did not cause significant nanoparticle size change. By contrast, with the anti-EGFR-GNP probe, significant particle size increase was observed when anti-Stat3 or anti-Src antibody was added to the assay solution (Fig. 4B(ii), anti-
Stat3, Src). A smaller size increase was observed from anti-EGFR antibody (Fig. 4B(ii), anti-EGFR) and the rabbit IgG (Fig. 4B(ii), IgG). Per the assay principle (see Fig. S2), the substantial particle size increases observed from the addition of anti-Stat 3 or anti-Src antibody to the assay solution can only be explained by the presence of EGFR in complex with Stat3 and Src in the nuclear extract. EGFR was specifically bound to the nanoparticle immunoprobes, bringing along the Stat3 and Src proteins to the nanoparticle surface, and the subsequent incubation of this assay solution with anti-Stat 3 or anti-Src antibody led to further increase of the nanoparticle size. Furthermore, it appears that there is an equal amount of Stat3 and Src proteins in the EGFR, Src, and Stat3 complex. In multiple assays conducted so far, the size increase caused by anti-Src antibody is always just slightly lower than the case of anti-Stat 3 antibody. Src is a smaller protein (60 KDa) than Stat3 (89-90 KDa). Therefore, with the binding of the same amount of antibody to the protein complex-GNP conjugates, the particle size should be just slightly smaller in the case of anti-Src assay solution than anti-Stat3 assay solution.

Compared to anti-Stat3 or anti-Src antibody, the particle size increase upon addition of anti-EGFR antibody to the assay solution is much smaller, only slightly higher than the non-specific rabbit IgG. This is explained by the fact that Stat 3 and Src are located on the surface of the nanoparticle-bound EGFR protein, therefore, block the binding of anti-EGFR antibody to the bound EGFR proteins. The effectiveness of polyclonal anti-EGFR antibody with the EGFR protein in the sample was confirmed in a different 
experiment (data not shown). This result indirectly suggested that the EGFR protein detected by the nanoparticle immunoprobe from the nuclear extract exists as a complex with Stat3 and Src protein.

Detection of EGFR, Src and Stat3 heterocomplex by immunofluorescence with laser-scanning confocal microscopy

Immunofluorescence with laser-scanning confocal microscopy allowed visualization of the intracellular distribution and localization patterns of EGFR, Src and Stat3. Immunofluorescence with laser-scanning confocal microscopy confirmed the localization patterns and showed that in contrast to the negative staining (Fig. 5B(i), left panel), EGFR appearance is punctuate (red) at the plasma membrane, and in the cytoplasm and the nucleus (Fig. 5B(i), single). Similar localizations for Src (blue) and Stat3 (green) were observed, but with greater presence in the nucleus (Fig. 5B(i), single). There are stainings for colocalization of EGFR and Src (magenta, see arrows), EGFR and Stat3 (yellow, see arrows), Src and Stat3 (cyan, see arrows), and of all three entities (pale yellow/white, see white arrows) at the plasma membrane, cytoplasm, perinuclear and nuclear spaces (Fig. 5B(i), merge).

A
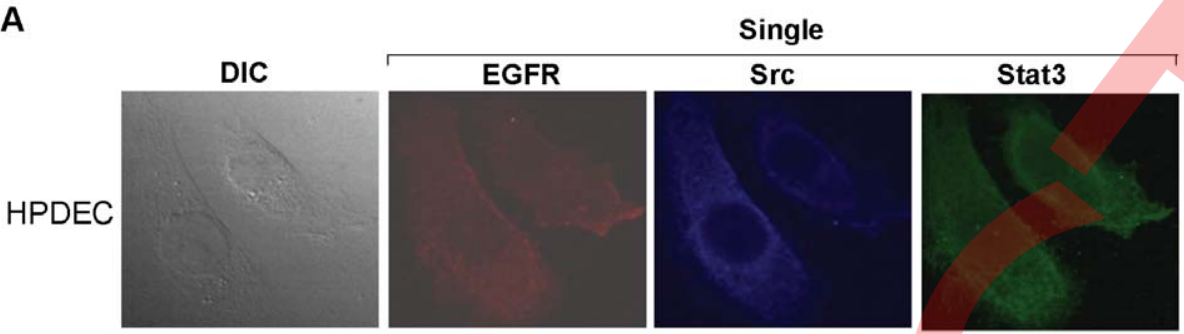

Merge

$\mathbf{B}_{\text {(i) }}$
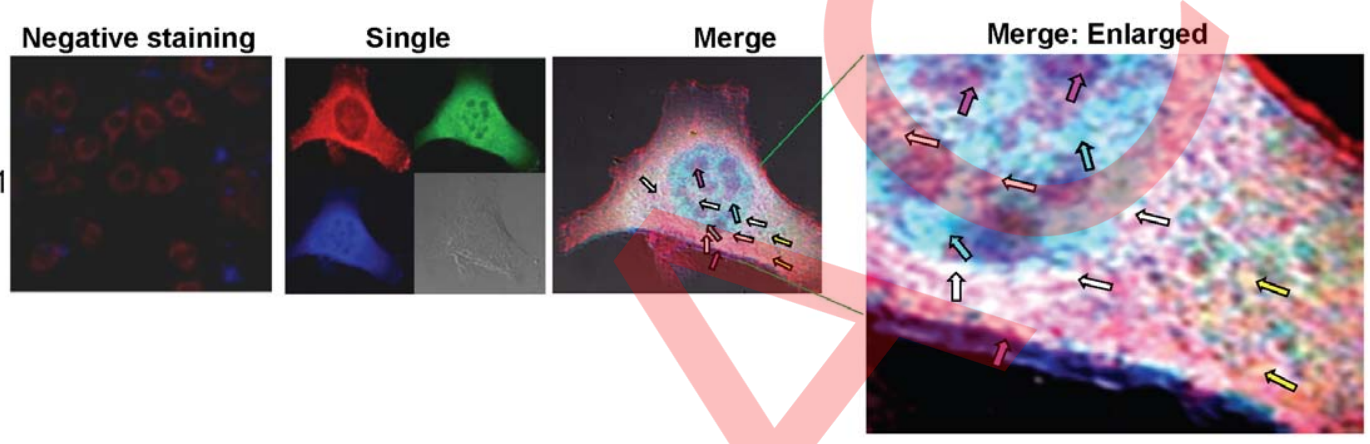

(ii) Single
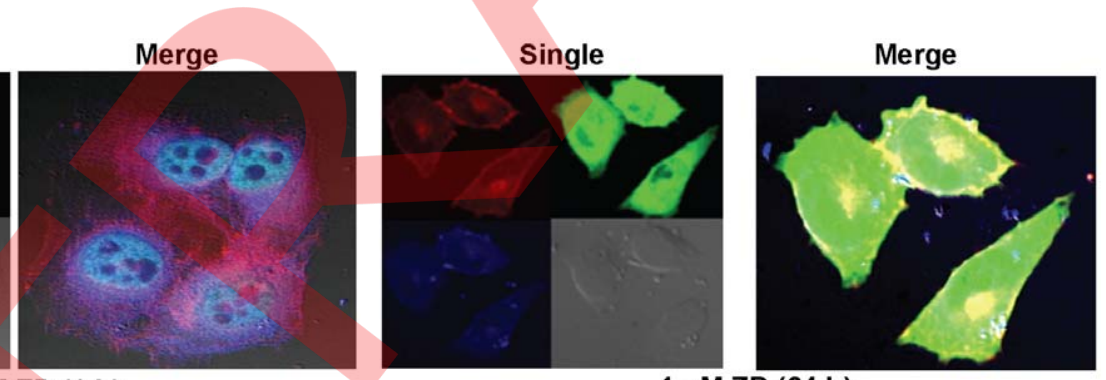

$1 \mu \mathrm{M} \mathrm{ZD}(1 \mathrm{~h})$

(iii) Single

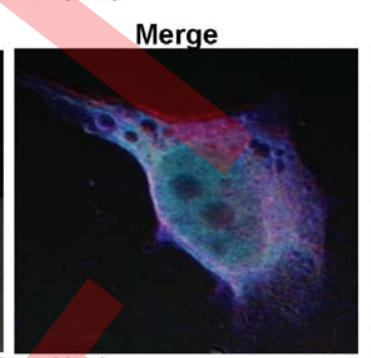

$100 \mathrm{nM}$ Das $(1 \mathrm{~h})$

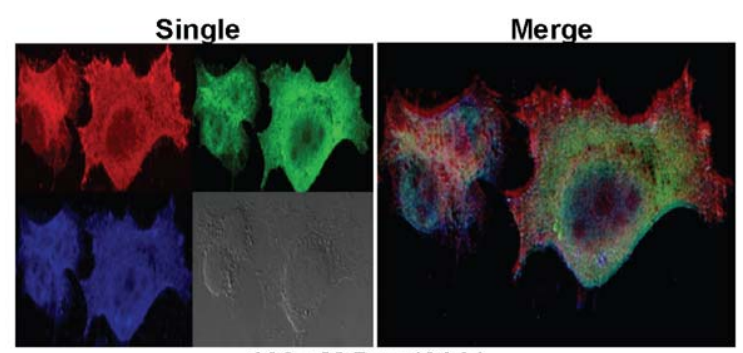

$100 \mathrm{nM}$ Das $(24 \mathrm{~h})$

Figure 5. Immunofluorescence with laser-scanning confocal microscopy of EGFR, Src and Stat3 association in HPDEC or Panc-1 cells. Cultured normal human pancreatic duct epithelial cells (HPDEC) (A) or pancreatic cancer, Panc-1 cells (B) were fixed, stained with primary antibodies against EGFR, Src and Stat3 and their corresponding secondary antibodies, ALexaFLuor405 (goat anti-mouse, EGFR, red), AlexaFluor488 (donkey anti-rabbit, Src, blue) and AlexaFluor546 (goat anti-rat, Stat3, green) and analyzed by laser-scanning confocal microscopy for localization (single) and colocalization (merge) studies of EGFR (red), Src (blue) and Stat3 (green) and the effects of treatment (i) without or (ii) with ZD1839 (ZD) or (iii) Dasatinib (Das) for the indicated times. Confocal images were collected using Leica TCS SP5 microscopes; Cyan, magenta, yellow and white/ pale yellow arrows denote merged colors; single, one color capture, merged, three-color capture. Data are representative of 3 independent studies. doi:10.1371/journal.pone.0019605.g005 
These studies confirm previous reports of the association of EGFR and Stat 3 in similar intracellular spaces $[6,10]$. Importantly, the data show for the first time the presence of a heteromeric EGFR, Src and Stat 3 complex in the nucleus, as observed by the co-IP and immunoblotting analyses, and confirmed by DANS/DLS analysis. These data contrast the results observed for the normal human pancreatic duct epithelial cells (HPDEC) (Fig. 5A). Immunofluorescence staining with laser-scanning confocal microscopy analysis of HPDEC shows a homogeneous distribution of EGFR (red), Src (blue) and Stat3 (green), all of which are strongly outside of the nucleus, with little evidence of co-localization (Fig. 5A).

Visualization of the intracellular distribution patterns of EGFR, Src and Stat3 upon inhibition of EGFR or Src tyrosine kinase by immunofluorescence with laser-scanning confocal microscopy indicated that as with the co-IP studies (Fig. 3B), the inhibition of Src or EGFR tyrosine kinase activity alone did not completely eliminate the complex formation. However, Src or EGFR inhibition disrupted the localization patterns. Data shows EGFR, Src and Stat3 distribution in the cytoplasm and the nucleus following 1- or 24-h treatment with EGFR or Src inhibitor (Fig. 5B(ii) and (iii), $1 \mathrm{~h}$, and $24 \mathrm{~h}$, single). Nuclear EGFR levels are diminished, but not abolished (Fig. 5B(ii), single). Thus, EGFR, Src and Stat3 remain localized in the nucleus following tyrosine kinase inhibition. Results further showed persistent EGFR and Src (magenta), and Src and Stat3 (cyan) associations in both the nucleus and cytoplasm following treatment for $1 \mathrm{~h}$ with EGFR or Src inhibitor (Fig. 5B(ii) and (iii), $1 \mathrm{~h}$ ), similar to the data in Figure 3B (1 h). Furthermore, a nuclear EGFR and Stat3 association (yellow) is detected upon 24-h treatment with the EGFR inhibitor (Fig. 5B(ii), $100 \mathrm{nM} \mathrm{ZD,} 24 \mathrm{~h}$ ), while nuclear associations of EGFR and Src (magenta), and of Src and Stat3 (cyan) are detected following treatment with Src inhibitor for $24 \mathrm{~h}$ (Fig. 5B(iii), $100 \mathrm{nM}$ Das, $24 \mathrm{~h}$ ). The moderate differences in the observed patterns of complex formation between the co-IP (Fig. 3B) and confocal data may be due to the sensitivity differences between the two approaches. Overall, the findings are consistent with the co-IP data (Fig. 3B) in showing that the inhibition of the kinase activity of EGFR or Src alone is insufficient to completely disrupt all the proteins from the complex.

\section{EGFR, Src and Stat3 heteromeric complex regulates the c-Myc gene expression}

In our previous work, we showed that pancreatic cancer cells were insensitive to the inhibition of EGFR, Src or Stat3 activity alone, in parallel with the observation that the expression of c-Myc was also refractory to the inhibition of EGFR, Src or Stat3 alone, while the concurrent inhibition of aberrant Stat3 activity together with EGFR or Src inhibition strongly suppressed c-Myc expression and induced stronger antitumor cell effects [16]. Those findings suggest a complex regulation of c-Myc induction, which might support the cancer phenotype. Previous report identified only that the nuclear EGFR and Stat 3 complex in breast cancer cells induced specific genes, including inducible nitric oxide synthase (iNOS) [10]. Studies were performed to assess the functional significance of the heteromeric complex, particularly in the context of the induction of the c-Myc gene in pancreatic cancer cells. Chromatin immunoprecipitation (ChIP) analysis was pursued to assess the association of the c-Myc promoter with the heteromeric complex. PCR amplification of DNA fragment using a primer against the c-Myc gene promoter and agarose gel electrophoresis showed that each of the anti- EGFR, Src, or Stat3 antibody-chromatin DNA immunoprecipitate contained the c-Myc gene (Fig. 6A(i), EGFR, Src and Stat3). To further confirm this finding, we pursued a modified sequential immunoprecipitation analysis similar to the one performed in Figure 2B in the
$A_{\text {(i) }}$

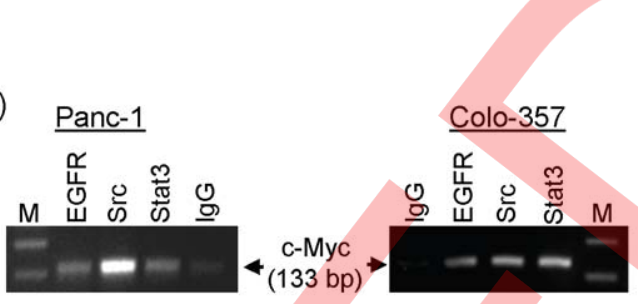

(ii)

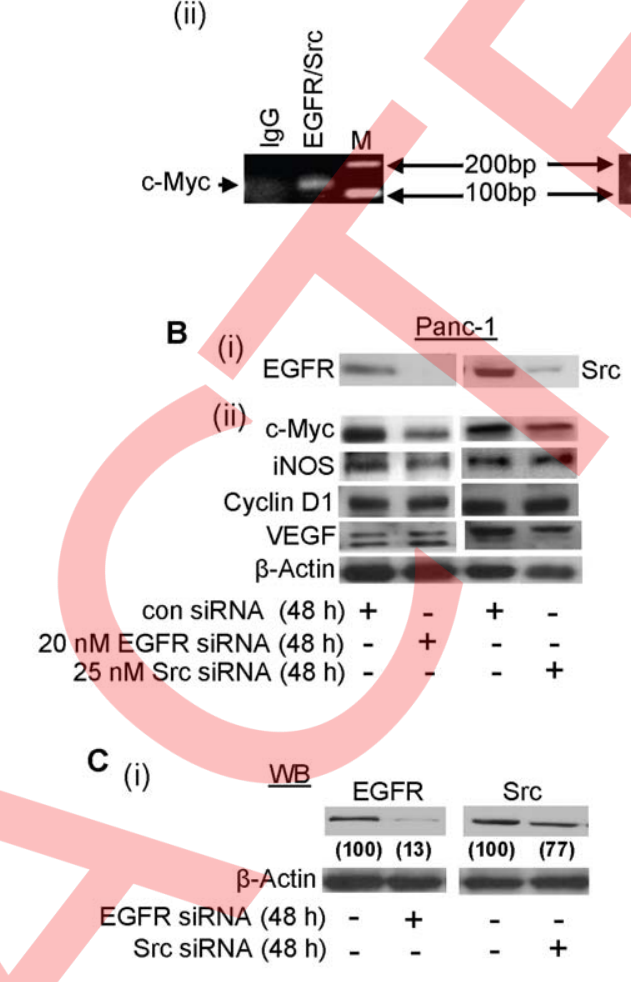

(ii) WB

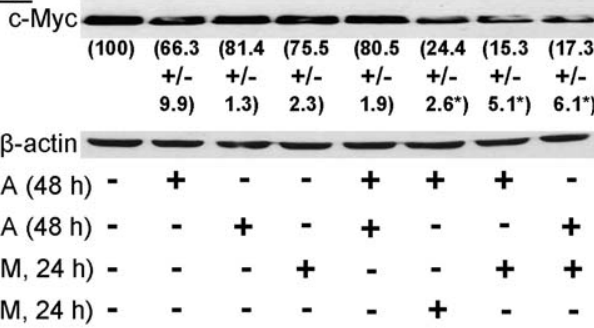

Figure 6. Chromatin immunoprecipitation assay and Western blotting analysis of c-Myc, iNOS, Cyclin D1, and VEGF expression in Panc-1 and Colo-357 cells. (A), Agarose gel electrophoresis of the Polymerase Chain Reaction (PCR)-amplified c-Myc gene fragment from the chromatin DNA precipitated with antibody against EGFR, Src, or Stat3, or with the non-specific IgG; and (B and $C$ ), Immunoblotting analysis of whole-cell lysates probing for EGFR or Src (B(i) and C(i)) or CMyc, iNOS, Cyclin D1 or VEGF (B(ii) and C(ii)), and the effects of siRNA knockdown of EGFR (EGFR siRNA), Src (Src siRNA) or control (con) siRNA, or S3I-201 or Das). Bands corresponding to proteins or c-Myc gene in gel are shown; $M$, molecular weight marker, EGFR/Src, sequential immunoprecipitation with anti-EGFR and then anti-Src antibody. Data are representative of 3 independent studies, and values are mean and s.d of 3 independent studies; ${ }^{*} p-<0.01$.

doi:10.1371/journal.pone.0019605.g006

context of a ChIP assay, as previously reported [10]. In the sequential ChIP assay in which EGFR chromatin immunecomplex was subjected to a second immunoprecipitation using anti-Src antibody and analyzed by PCR amplification and agarose gel electophoresis, we similarly detected the presence of the c-Myc gene 
(Fig. 6A(ii), EGFR/Src). By contrast, PGR analysis showed no appreciable detection of TWIST, a gene previously reported to be EGFR target [22] in the EGFR chromatin immunecomplex, while the gene was detected in the Stat 3 and Src chromatin-immunoprecipitates (Fig. S3). These differences may be tumor cell-type dependent. To verify the specificity of the immunoreagents, the non-specific IgG was similarly used in the ChIP assay, and subsequent PCR analysis showed no detectable levels of the c-Myc gene (Fig. 6A, IgG). Taken together with the sequential immunoprecipitation data in Figure 2B), these studies demonstrate that EGFR, Src and Stat3 form a detectable heteromeric complex that is associated with the expression of $c-m y c$ in pancreatic cancer cells.

To further study the potential involvement of the heteromeric EGFR, Src and Stat3 complex in the regulation of genes, we performed immunoblotting analysis of known regulated genes, including c-Myc. Results showed moderate or no significant change in the expression of c-Myc, Cyclin D1, iNOS, and VEGF upon the siRNA knockdown of EGFR or Src alone (Fig. 6B). By contrast, the concurrent knockdown of EGFR with Stat3 inhibition (by S3I-201) [25], or the concurrent knockdown of Src with Stat3 inhibition, or the concurrent EGFR knockdown with Src inhibition (by Das) resulted in a strong suppression of cMyc expression (Fig. 6C(ii)). The bands corresponding to the expression levels of c-Myc were quantified, analyzed by ImageQuant, and represented as percent of control (Fig. 6C(ii), \% numbers in parenthesis). Results show over $76 \%$ suppression of cMyc expression (Fig. 6C(ii)) following the concurrent modulation of any two of EGFR, Src and Stat3, except for the siRNA knockdown of EGFR and Src together, which only showed 20\% decrease. The moderate change in the c-Myc expression in response to the co-transfection with EGFR siRNA and c-Src siRNA may be due to the fact that we could only achieve partial knockdown of Src (Fig. 6C(i)), although EGFR is significantly suppressed by the EGFR siRNA (Fig. 6G(i)). In contrast, the use of the pharmacological inhibitor, Das, which strongly inhibits Src activity, in combination with siRNA knockdown of EGFR strongly suppressed c-Myc expression. These studies together suggest the possibility that the c-Myc gene is regulated by EGFR, Src and Stat3 complex in a manner that is susceptible to concurrent modulation of any two of the EGFR, Src and Stat3 proteins, but not to the inhibition of EGFR, Src or Stat3 alone.

\section{Discussion}

Aberrations in the EGFR, c-Src and Stat3 signaling pathways occur with a high frequency in many human cancers $[14,15,38,39,40,41]$ and are associated with poor prognosis. Notably, constitutively-active Stat3 induces dysregulation of gene expression, contributing to the altered gene expression profile that is a hallmark of cancer. The details of aberrantly-active Stat3mediated dysregulation of gene expression continue to be elucidated and the initial studies indicate the mechanisms are more complex. The present studies strongly suggest that in forming a nuclear, transcriptionally-active EGFR, Src, Stat3 heteromeric complex, the EGFR and Src proteins cooperate with Stat 3 to promote the altered gene expression. Such cooperation between Stat 3 and other proteins for the transcriptional induction of genes has similarly been observed in other systems. Specifically, Stat3 cooperates with NF- $\mathrm{kB}$ to induce certain genes $[42,43]$. Furthermore, there have been reports of a nuclear EGFR pathway $[8,9]$, in which a nuclear EGFR-Stat3 complex promotes the induction of iNOS in breast cancer cells [10]. These reports together with our present findings indicate the complicated nature of the mechanisms by which aberrantly-active Stat3 might dysregulate gene expression in cancer cells. In this context, the present studies extend our earlier report [16] in suggesting that nuclear heteromeric EGFR, Src and Stat3 complex regulates the c-Myc gene in pancreatic cancer cells. It is likely that other genes may by induced by the EGFR, Src and Stat 3 nuclear complex and we note that iNOS, VEGF, and Cyclin D1 are reported to be induced by the EGFR/Stat 3 complex or Stat3 $[10,44,45]$ and could well be candidates for the regulation by the EGFR, Src and Stat3 complex. Taken together, these studies provide a novel mechanism for the de-regulation of gene expression in cancer cells.

The observation that the EGFR, Src and Stat 3 complex is also detected in the cytoplasm raises the possibility that it is formed extra-nuclear and transported into the nuclear space. Previous reports have described inherent EGFR nuclear localization mechanisms, including facilitation via the endosomal sorting machinery and the interaction with importins $\alpha 1 / \beta 1$ [31], and several nuclear translocation mechanisms have also been proposed for Stat3, including EGFR-mediated endocytosis [6,31]. Although any of these processes could facilitate the nuclear translocation of the heteromeric EGFR, Src, Stat3 complex should it be formed outside of the nucleus, the present data excludes the possibility that EGFR-mediated endocytosis is involved. Whether the other nuclear translocation pathways proposed for Stat3, including the utilization of the Ran-GTPase [4], Sec61 translocon [46], and chaperoning by MgcRacGAP [5] are involved in promoting the nuclear transport of the complex remains to be studied. Our findings also do not preclude the assembly of the complex de novo in the nucleus. Present data also suggest that only a portion of the intracellular EGFR, Src and Stat3 protein pools are utilized in the formation of the nuclear complex, raising the possibility that there may be diverse cellular pools of EGFR, Src, or Stat3 with different accessibility limitations. There also could be different pools of preassociated complexes of the three proteins, a possibility that will be consistent with the report that cytoplasmic Stat3 exists as complexes with accessory scaffolding proteins [47]. Such preformed complexes would not only facilitate the signal induction [48], but may also serve to stabilize the proteins.

The incidence of signaling cross-talk has long been known, and the associations of EGFR with Src [49], Src with Stat3 [20,50], and EGFR with Stat3 [26,27] at the plasma membrane and the perinuclear space [6] have been reported. Specifically, Stat3 binds to pY1068 and pY1086 motifs of EGFR [26,27], while Src binds to Y845EGFR. In cancer cells, aberrant Stat3 activation is promoted by hyperactive protein tyrosine kinases, including EGFR and Src $[15,40,41]$, and evidence has indicated that c-Src phosphorylates Y845EGFR, Y1068EGFR, and Y845EGFR motifs in pancreatic cancer cells $[16,27,51]$. Present data reveals that the pY1068EGFR and pY1086EGFR motifs and the Stat3 SH2 domain amino acid residues 588-615 are essential for EGFR-Stat3 interaction within the context of the heteromeric EGFR, Src and Stat3 complex. It remains to be determined what the exact configuration is for the heteromeric complex. Also, the present data does not exclude the possibility that other accessory proteins could be present in the complex together with EGFR, Src and Stat3.

We had recently reported about the functional cooperation between EGFR, Src and Stat3 in promoting and supporting pancreatic cancer, wherein the cancer phenotype and the expression of $\mathrm{c}-\mathrm{Myc}$ in the cancer cells were both insensitive to the inhibition of EGFR, Src or Stat3 alone [16]. While it is possible that the c-Myc gene may be regulated cooperatively by EGFR, Src and Stat3 through mechanisms independent of each other, our study also raises the possibility of a complex transcriptional regulation by mechanisms that involve the nuclear EGFR, Src and Stat3 heteromeric complex. 


\section{Supporting Information}

Text S1 Detail description of Dynamic Light Scattering (DLS).

(DOC)

Figure S1 Immunoblotting analysis of EGFR, Src, Stat3, and RHA from Panc-1, MDA-MB-231, or A549 cells. Immunoblotting analysis of (A and B) fractions collected from gel filtration chromatographic analysis of Panc-1 cell lysates probing for EGFR, Src, Stat3,or RHA (A), or EGFR immunecomplex from the pooled fractions 23-27 probing for Stat3, Src or EGFR (B), or $(\mathrm{C})$ immunecomplexes of EGFR or Stat3 from nuclear extracts of human breast cancer, MDA-MB-231 or non-small cell lung cancer, A549 cells probing for EGFR, Stat3 and Src. Bands corresponding to proteins in gel are shown; Data are representative of 2 independent studies.

(TIF)

Figure S2 The principle of DANS technology (Detection and Analysis through Nanoparticle Sizing) for protein complex detection and binding partner analysis.

(TIF)

\section{References}

1. Ulrich A, Schlessinger J (1990) Signal transduction by receptors with tyrosine kinase activity. Cell 61: 203-212.

2. Schlessinger J (2000) Cell signaling by receptor tyrosine kinases. Cell 103: 211-225.

3. Darnell JE (2005) Validating Stat3 in cancer therapy. Nat Med 11: 595-596.

4. Liu L, McBride KM, Reich NG (2005) STAT3 nuclear import is independent of tyrosine phosphorylation and mediated by importin-alpha3. Proc Natl Acad Sci USA 102: 8150-8155.

5. Kawashima T, Bao YC, Minoshima Y, Nomura Y, Hatori T, et al. (2009) A Rac GTPase-activating protein, MgcRacGAP, is a nuclear localizing signalcontaining nuclear chaperone in the activation of STAT transcription factors. Mol Cell Biol 29: 1796-1813.

6. Bild AH, Turkson J, Jove R (2002) Cytoplasmic transport of Stat3 by receptormediated endocytosis. Embo J 21: 3255-3263.

7. Sehgal PB, Guo GG, Shah M, Kumar V, Patel K (2002) Cytokine signaling: STATS in plasma membrane rafts. J Biol Chem 277: 12067-12074.

8. Lo HW, Hsu SC, Hung MC (2006) EGFR signaling pathway in breast cancer: from traditional signal transduction to direct nuclear translocation. Breast Cancer Res Treat 95: 211-218.

9. Lin SY, Makino K, Xia W, Matin A, Wen Y, et al. (2001) Nuclear localization of EGF receptor and its potential new role as a transcription factor. Nat Gell Biol 3: 802-808.

10. Lo HW, Hsu SC, Ali-Seyed M, Gunduz M, Xia W, et al. (2005) Nuclear interaction of EGFR and STAT3 in the activation of the iNOS/NO pathway. Cancer Cell 7: 575-589.

11. Korc M, Meltzer P, Trent J (1986) Enhanced expression of epidermal growth factor receptor correlates with alterations of chromosome 7 in human pancreatic cancer. Proc Natl Acad Sci U S A 83: 5141-5144.

12. Trevion JG, Summy JM, Lesslie DP, Parikh NU, Hong DS, et al. (2006) Inhibition of SRC expression and activity inhibits tumor progression and metastasis of human pancreatic adenocarcinoma cells in an orthotopic nude mouse model. Am J Pathol 168: 962-972.

13. Trevino JG, Gray MJ, Nawrocki ST, Summy JM, Lesslie DP, et al. (2006) Src activation of Stat3 is an independent requirement from NF-kappaB activation for constitutive IL-8 expression in human pancreatic adenocarcinoma cells. Angiogenesis 9: 101-110

14. Scholz ASH, Detjen KM, Peters M, Welzel M, Hauff P, et al. (2003) Activated signal transducer and activator of transcription 3 (STAT3) supports the malignant phenotype of human pancreatic cancer. Gastroenterology 125: 891-905.

15. Turkson J (2004) STAT proteins as novel targets for cancer drug discovery. Expert Opin Ther Targets 8: 409-422.

16. Jaganathan S, Yue P, Turkson J (2010) Enhanced sensitivity of pancreatic cancer cells to concurrent inhibition of aberrant signal transducer and activator of transcription 3 and epidermal growth factor receptor or Src. J Pharmacol Exp Ther 333: 373-381.

17. Turkson J, Ryan D, Kim JS, Zhang Y, Chen Z, et al. (2001) Phosphotyrosyl peptides block Stat3-mediated DNA-binding activity, gene regulation and cell transformation. J Biol Chem 276: 45443-45455.

18. Ouyang H, Mou LJ, Luk C, Liu N, Karaskova J, et al. (2000) Immortal human pancreatic duct epithelial cell lines with near normal genotype and phenotype. Am J Pathol 157: 1623-1631.

19. Turkson J, Bowman T, Garcia R, Caldenhoven E, De Groot RP, et al. (1998) Stat3 activation by Src induces specific gene regulation and is required for cell transformation. Mol Cell Biol 18: 2545-2552.
Figure S3 Chromatin Immunoprecipitation assay of TWIST expression in Panc-1 cells. Agarose gel electrophoresis of the Polymerase Chain Reaction (PCR)-amplified TWIST gene fragment from the chromatin DNA precipitated with antibody against EGFR, Src, or Stat3, or with the non-specific IgG; M, molecular weight marker; Data are representative of 2 independent studies.

(TIF)

\section{Acknowledgments}

We thank all colleagues and members of our laboratory for the stimulating discussions and the University of Central Florida Confocal Microscopy Group for the assistance with the confocal imaging work.

\section{Author Contributions}

Conceived and designed the experiments: SJ QH JT. Performed the experiments: SJ PY DCP JB. Analyzed the data: SJ QH JT. Contributed reagents/materials/analysis tools: QH JT. Wrote the paper: SJ QH JT.

20. Zhang Y, Turkson J, Carter-Su C, Smithgall T, Levitzki A, et al. (2000) Activation of Stat3 in v-Src Transformed Fibroblasts Requires Cooperation of Jak1 Kinase Activity. J Biol Chem 275: 24935-24944.

21. Siddiquee K, Glenn M, Gunning P, Katt WP, Zhang S, et al. (2007) An oxazolebased small-molecule Stat 3 inhibitor modulates Stat3 stability and processing and induces antitumor cell effects. ACS Chem Biol 2: 787-798.

22. Lo HW, Hsu SC, Xia W, Cao X, Shih JY, et al. (2007) Epidermal growth factor receptor cooperates with signal transducer and activator of transcription 3 to induce epithelial-mesenchymal transition in cancer cells via up-regulation of TWIST gene expression. Cancer Res 67: 9066-9076.

23. DeArmond D, Brattain MG, Jessup JM, Kreisberg J, Malik S, et al. (2003) Autocrine-mediated ErbB-2 kinase activation of STAT3 is required for growth factor independence of pancreatic cancer cell lines. Oncogene 22: 7781-7795.

24. Huo L, Wang YN, Xia W, Hsu SC, Lai CC, et al. (2010) RNA helicase A is a DNA-binding partner for EGFR-mediated transcriptional activation in the nucleus. Proc Natl Acad Sci USA 107: 16125-16130.

25. Siddiquee K, Zhang S, Guida WC, Blaskovich MA, Greedy B, et al. (2007) Selective chemical probe inhibitor of Stat3, identified through structure-based virtual screening, induces antitumor activity. 1: Proc Natl Acad Sci U S A 104: 7391-7396.

26. Coffer PJ, Kruijer W (1995) EGF receptor deletions define a region specifically mediating STAT transcription factor activation. Biochem Biophys Res Commun 210: 74-81.

27. Shao H, Cheng HY, Cook RG, Tweardy DJ (2003) Identification and characterization of signal transducer and activator of transcription 3 recruitment sites within the epidermal growth factor receptor. Cancer Res 63: 3923-3930.

28. Darnell JE, Jr. (1997) STATs and gene regulation. Science 277: 1630-1635.

29. Zhao W, Jaganathan S, Turkson J (2010) A cell permeable Stat3 SH2 domain mimetic inhibits Stat 3 activation and induces antitumor cell effects in vitro. J Biol Chem, 2010 Aug 31. [Epub ahead of print].

30. Hsu SC, Hung MC (2007) Characterization of a novel tripartite nuclear localization sequence in the EGFR family. J Biol Chem 282: 10432-10440.

31. Lo HW, Ali-Seyed M, Wu Y, Bartholomeusz G, Hsu SC, et al. (2006) Nuclearcytoplasmic transport of EGFR involves receptor endocytosis, importin betal and CRM1. J Cell Biochem 98: 1570-1583.

32. Huo Q (2010) Protein complexes/aggregates as potential cancer biomarker revealed by a nanoparticle aggregation immunoassay. Colloids Surf B 78: 259-265.

33. Bognadovic J, Huo Q (2010) NanoDLSay: NanoDLSay: a new platform technology for biomolecular detection and analysis using gold nanoparticle probes coupled with dynamic light scattering. Proceedings of SPIE 7674: In press.

34. Jans H, Liu X, Austin L, Maes G, Huo Q (2009) Dynamic light scattering as a powerful tool for gold nanoparticle bioconjugation and biomolecular binding study. Anal Chem 81: 9425-9432.

35. Liu X, Huo Q (2009) A washing-free and amplification-free one-step homogeneous assay for protein detection using gold nanoparticle probes and dynamic light scattering. J Immunol Method 349: 38-44.

36. Liu X, Dai Q Austin L, Coutts J, Knowles G, et al. (2008) A One-step homogeneous immunoassay for cancer biomarker detection using gold nanoparticle probes coupled with dynamic light scattering. J Am Chem Soc 130: $2780-2782$.

37. Dai Q Liu X, Coutts J, Austin L, Huo Q (2008) A one-step highly sensitive method for DNA detection using dynamic light scattering. J Am Chem Soc 130: 8138-8139. 
38. Thelemann A, Petti F, Griffin G, Iwata K, Hunt T, et al. (2005) Phosphotyrosine signaling networks in epidermal growth factor receptor overexpressing squamous carcinoma cells. Mol Cell Proteomics 4: 356-376.

39. Jimeno A, Tan AC, Coffa J, Rajeshkumar NV, Kulesza P, et al. (2008) Coordinated epidermal growth factor receptor pathway gene overexpression predicts epidermal growth factor receptor inhibitor sensitivity in pancreatic cancer. Cancer Res 68: 2841-2849.

40. Turkson J, Jove R (2000) STAT proteins: novel molecular targets for cancer drug discovery. Oncogene 19: 6613-6626.

41. Yue P, Turkson J (2009) Targeting STAT3 in cancer: how successful are we? Expert Opin Investig Drugs 18: 45-56.

42. Grivennikov SI, Karin M (2010) Dangerous liaisons: STAT3 and NF-kappaB collaboration and crosstalk in cancer. Cytokine Growth Factor Rev 21: 11-19.

43. Yang J, Liao X, Agarwal MK, Barnes L, Auron PE, et al. (2007) Unphosphorylated STAT3 accumulates in response to IL-6 and activates transcription by binding to NFkappaB. Genes Dev 21: 1396-1408.

44. Sinibaldi N, Wharton W, Turkson J, Bowman T, Pledger WJ, et al. (2000) Induction of p21WAF1/CIP1 and cyclin D1 expression by the Src oncoprotein in mouse fibroblasts: role of activated STAT3 signaling. Oncogene 19: 5419-5427.
45. Niu G, Wright KL, Huang M, Song L, Haura E, et al. (2002) Constitutive Stat3 activity up-regulates VEGF expression and tumor angiogenesis. Oncogene 21: 2000-2008.

46. Liao H-J, Carpenter G (2007) Role of Sec61 translocon in EGF receptor trafficking to the nucleus and gene expression. Cell 18: 1064-1072.

47. Ndubuisi MI, Guo GG, Fried VA, Etlinger JD, Sehgal PB (1999) Cellular physiology of STAT3: Where's the cytoplasmic monomer? J Biol Chem 274: 25499-25509.

48. Guo GG, Patel K, Kumar V, Shah M, Fried VA, et al. (2002) Association of the Chaperone Glucose-Regulated Protein 58 (GRP58/ER-60/ERp57) with Stat3 in Cytosol and Plasma Membrane Complexes. J Interferon Cytokine Res 22: 555-563.

49. Biscardi JS, Maa MC, Tice DA, Cox ME, Leu TH, et al. (1999) c-Src-mediated phosphorylation of the epidermal growth factor receptor on Tyr845 and Tyr1101 is associated with modulation of receptor function. J Biol Chem 274: 8335-8343.

50. Cao X, Tay A, Guy GR, Tan YH (1996) Activation and association of Stat3 with Src in v-Src-transformed cell lines. Mol Cell Biol 16: 1595-1603.

51. Tice DA, Biscardi JS, Nickles AL, Parsons SJ (1999) Mechanism of biological synergy between cellular Src and epidermal growth factor receptor. Proc Natl Acad Sci U S A 96: 1415-1420.

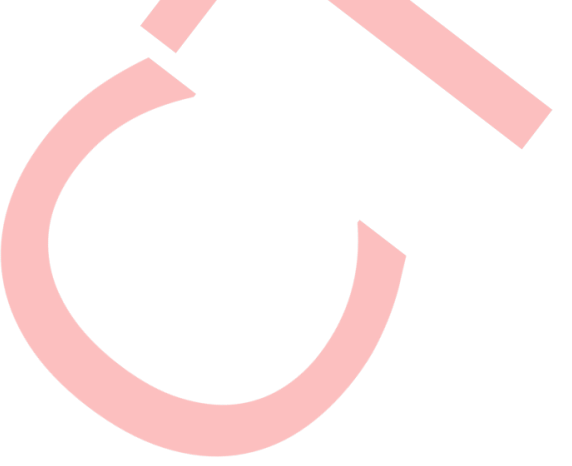

